\section{Cells and Cell-Interactions that Guide Motor Axons in the Developing Chick Embryo}

\author{
Kathryn W. Tosney
}

\section{Summary}

A considerable challenge confronts any developing neuron. Before it can establish a functional and specific connection, it must extend an axon over tens and sometimes hundreds of microns through a complex and mutable environment to reach one out of many possible destinations. The field of axonal guidance concerns the control of this navigation process. To satisfactorily identify the cell interactions and molecular mechanisms that mediate axonal guidance, it is essential to first identify the pertinent cell populations. Embryonic surgeries have provided solid information on which tissues are critical and which are irrelevant to the navigation of motor axons within the chick embryo. The gross anatomical nerve pattern is established as axons respond to both positive (path) and negative (barrier) tissue environments. Analysis of the interactions of motoneurons with these tissues reveals that several cellular interactions - chemotaxis, substratum preference, and perhaps contact paralysis - are important to the common patterns of motor axon advance. Axons simultaneously interact with population-specific cues that have begun to be identified on the tissue level.

\section{Introduction}

Axonal guidance cues that guide motor and sensory axons in the hindlimb region of the chick embryo can be usefully placed in two categories (see ref. 1). General cues, like a set of public highways, channel a variety of neural populations down common paths and thereby define the gross anatomical nerve patterns. For instance, motor and sensory neurons that will innervate a variety of targets within the limb extend together within common spinal nerve paths and then spread within a plexus region at the limb base before they enter common nerve trunk paths within the limb (Fig. 1). In contrast, specific cues, like signposts, direct each axonal population where possible paths diverge. For instance, motor axons specified to innervate dorsal limb muscles diverge into the dorsal rather than the ventral nerve trunk path at the limb base (motoneurons A in Fig. 1). The outgrowing axon must thus be responsive to two types of cues simultaneously; those cues that keep it within a common path, and those cues that direct it along a specific subset of the possible paths. It is the tip of the outgrowing axon, the growth cone, which possesses motile activity and responds to the guidance cues.

A first step in understanding how the general and specific guidance cues function is to define the guidance role of individual embryonic tissues. The surgical strategy for doing so is straightforward. If a tissue provides a specific cue, its deletion will alter the outgrowth pattern of only a single axonal population; if a tissue provides general cues, its deletion will alter the outgrowth patterns of several axonal populations. In this review, I address the guidance role of tissues that lie between the developing spinal cord and the limb of the chick embryo. The early architecture of these tissues is simple. On each side of the developing spinal cord lic blocks of tissue, the somites, which are serially repeated along the anterior-posterior axis; cach pair of somites defines an embryonic segment (Fig. 2). Each somite is composed of two major tissues just before the first axons extend (Fig. 3). The dorsal epithelial tissue, the

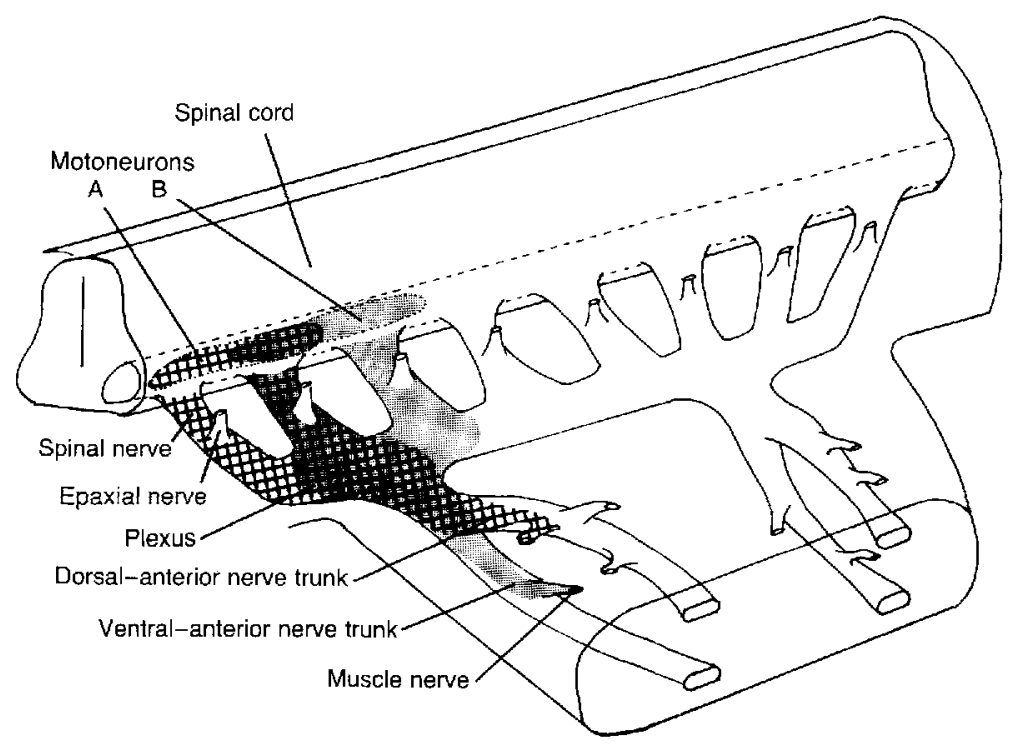

Fig. 1. Diagram of the nerve patterns in the hindlimb region of the chick embryo. Motoneurons that will innervate each muscle are grouped within the ventral spinal cord. Since several groups lie at each segmental level, axonal populations are mixed as they cxit from the spinal cord and several populations share spinal nerve, plexus and nerve trunk paths. General guidance cues delineate these common paths. Specific cues are required to explain the precise projection down subsets of paths. For instance, epaxial motor axons enter each spinal nerve path and then diverge dorsally to form the epaxial muscle nerve in each segment. In contrast, motoneurons for muscles A (cross-hatching) and B (stippling) extend axons through spinal nerve paths, diverge in the plexus region into the appropriate nerve trunk path, and then project specifically into single muscle nerves. Antcrior is to the left; dorsal is up. 


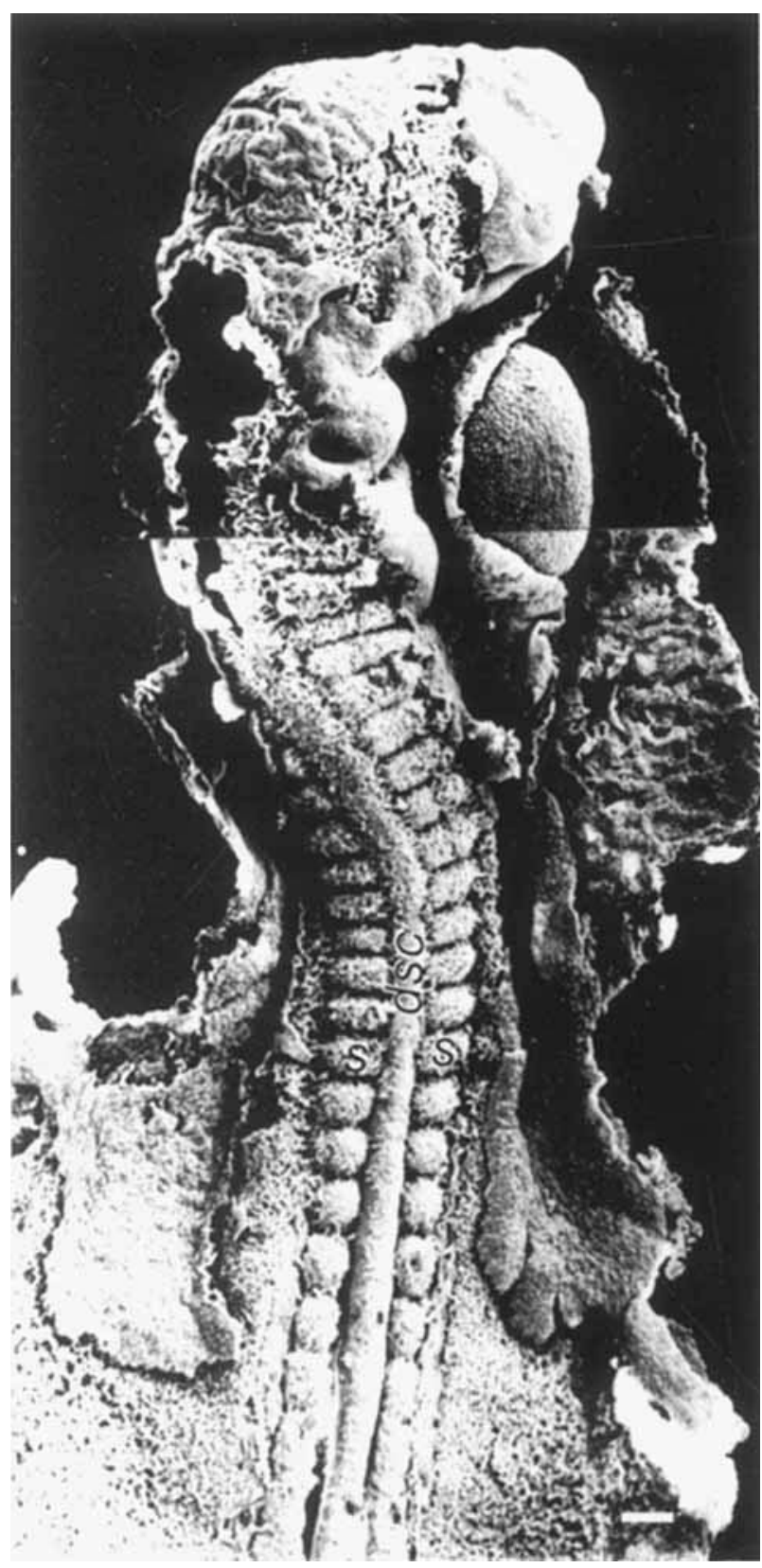

Fig. 2. Scanning electron micrograph of a 2 day embryo with the ectoderm removed shows the relatively simple architecture of the early embryo. Motor axons extending from the developing spinal cord will first encounter the somites, which are serially repeated blocks of tissue in the trunk of the embryo. Each pair of somites on the left and right side of the embryo defines an embryonic segment. Anterior is toward the top. Abbreviations: dsc, developing spinal cord; s, somites. Calibration bar $=100 \mu \mathrm{m}$.

dermamyotome, will later form the dermis and the epaxial muscles of the back; the ventral mesenchymal tissue, the sclerotome, will form vertebrae. Beyond the somites at the base of the limb lies the plexus mesenchyme and the prospective pelvic girdle (Fig. 3).

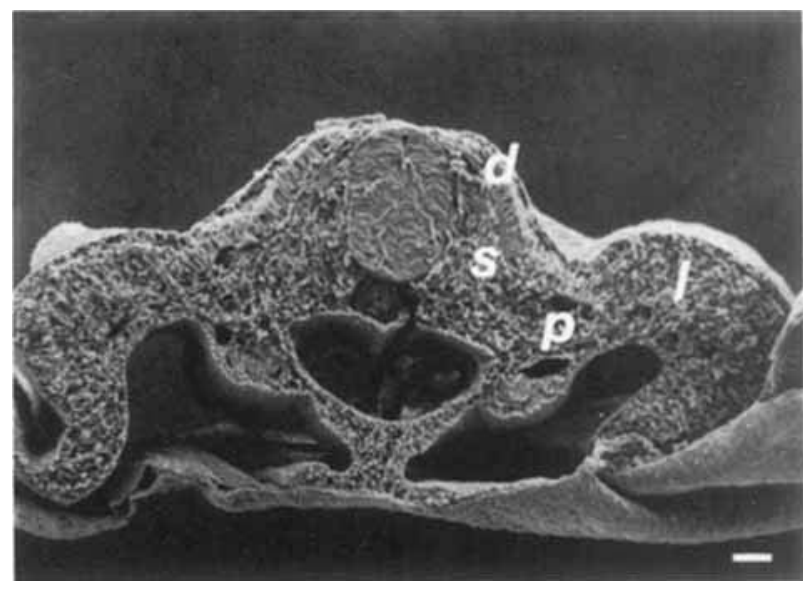

Fig. 3. Scanning electron micrograph of a cross-section shows the spatial relationship of cmbryonic tissues just before motor axons extend. Each somite has formed two distinct tissues. The dorsal dermamyotome is organized as an epithelium; it will later form dermis and epaxial muscle. The ventral somitic tissue, the sclerotome, is organized as a mesenchyme. Plexus mesenchyme lies at the base of the limb adjacent to the prospective pelvic girdle which is not yet histologically distinct. Dorsal is toward the top. Abbreviations: d, dermamyotome; s, sclerotome; $p$, plexus; 1 , limb. Calibration bar $=10 \mu \mathrm{m}$.

\section{A Tissue that Supplies a Specific Guidance Cue}

The search for tissues that provide specific guidance cues has been extensive. We know that cells within the plexus region supply multiple specific cues for the proper deployment of motor axons into the appropriate nerve trunk path (e.g., ref. 2) and that tissues within the limb provide short-range cues for the specific deployment of each motor population (e.g., ref. 3). In only one instance, however, has a tissue that is essential for the outgrowth of only a single motor population been identified. This is the dermamyotome, which forms the epaxial muscle.

Deletion of the dermamyotome shows that this muscle precursor is essential for the development of epaxial motor nerves ${ }^{(4)}$. Epaxial motor axons, unlike those motor axons destined for limb targets, normally diverge from the spinal nerve pathway while still within the somite and extend dorsally, directly toward the dermamyotome (Fig. 4, segments 1 and 5). When the dermamyotome is deleted from a segment, the epaxial motor axons in that segment extend only when dermamyotome is present in an adjacent segment. Moreover, these target-deprived axons extend in an oriented fashion toward the closest dermamyotome: those with neighboring dermamyotome to the anterior grow toward the anterior; those with neighboring dermamyotome to the posterior grow toward the posterior (Fig. 4, segments 2 and 4). In contrast, an epaxial nerve does not form when dermamyotome has also been deleted in both adjacent segments (Fig. 4, segment 3), as though the epaxial axons were too distant from dermamyotome to sense a cue required for 


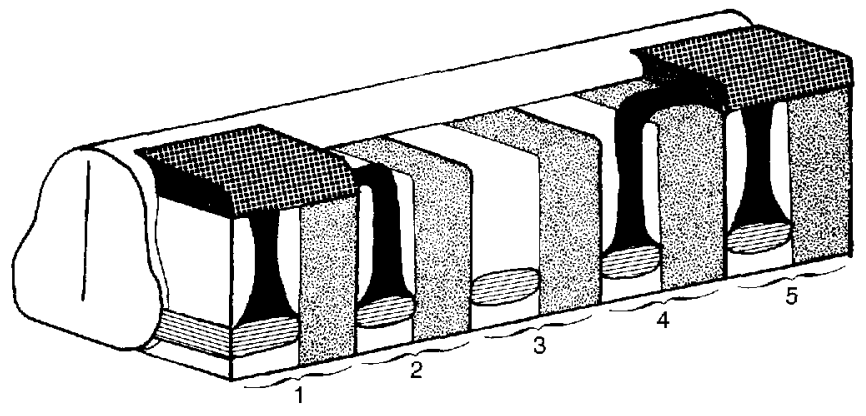

Fig. 4. Diagram of the effect of dermamyotome deletion on the patterns of motor axon outgrowth. Epaxial motor axons (black) normally extend dorsally from the spinal nerve path (horizontal lines) and enter the dermamyotome (squares) in the same segment, as shown in segments 1 and 5 . The segmental pattern is retained following dermamyotome delction in segments 2,3 , and 4 ; both epaxial and limb motor axons grow out in anterior (white) but not in posterior (stippling) sclerotome. However, epaxial molor axons exhibit a striking target dependence. In segments 2 and 4, epaxial motoneurons extend into the closest dermamyotome in an adjacent segment by taking a route that avoids the posterior sclerotome. In segment 3, epaxial motor nerves do not form, as though epaxial axons were too distant from any dermamyotome to detect an essential, stimulatory cue that diffuses from this tissue. Since motor axons project to their appropriate limb muscles despite the deletions, the dermamyotome provides cues that are essential only for the epaxial motor axons. Anterior is to the left; dorsal is up.

their outgrowth. While dermamyotomes are essential for the formation of epaxial muscle nerves, they are irrelevant to the outgrowth of limb motor axons which continue to project to their appropriate muscles in the absence of dermamyotomes. Therefore, the dermamyotome is essential only for the formation of epaxial motor innervation.

The cellular interactions that mediate the response of epaxial motoneurons to their developing muscle have yet to be unequivocally defined. The surgical evidence favors chemotaxis, an orientation toward the source of a diffusible cue, since epaxial motor nerves are not detected when dermamyotome is greater than $150 \mu \mathrm{m}$ away and axon growth is oriented toward dermamyotome that is within $150 \mu \mathrm{m}$. While these distances are clearly beyond the range of direct contact for growth cones within the spinal nerve pathway, it is not possible to rule out a contact-mediated mechanism with the surgical approach alone. For instance, epaxial axons might have transiently grown out but not been detected in operated embryos because they quickly retracted when they failed to contact dermamyotome. The finer temporal resolution provided by tissue culture approaches should show whether the dermamyotome is essential for epaxial motor nerve formation because it supplies a diffusible cue or because it stabilizes those epaxial axons that contact it.

\section{Three Pairs of Tissues that Provide General Cues}

Embryonic surgeries have now identified six tissues that provide general guidance cues for the outgrowth of motor and sensory axons. It is of particular interest that these tissues appear to function in pairs during axon guidance. Each pair provides a contrast between adjacent environments, one that acts as a path and one that acts as a barrier. I use the terms path and barrier because these terms do not presuppose a particular cellular or molecular mechanism. A barrier could be an impenetrable environment that precludes advance; it may consist of a completely non-permissive substratum or merely a less preferred substratum for growth cone advance; or, it may contain toxins or supply a specific substance that actively inhibits growth cone motility. Within the context of the embryo, a barrier is operationally defined as a tissue that axons will turn to avoid. Barrier as well as path function is essential to general guidance, just as walls are essential to the delineation of hallways within a building.

One set of path/barrier tissues within the somite is responsible for the development of segmentally patterned spinal nerves. It has long been known that the segmentally patterned somites impose their pattern on the axons that invade them, but even the spatial pattern of axon outgrowth relative to somites was unknown until recently. A segmental nerve pattern could theoretically develop because axons extend only between the segmented somites, or because axons extend only through the center of each somite. What actually happens is much more intriguing: axons grow only through the anterior half of each somite and do not extend into the posterior half ${ }^{(5)}$. The anterior and posterior halves of somites are not composed of obviously different tissue populations; axons must respond to some more subtle, intrinsic difference in the anterior and posterior halves of one or more somitic tissues.

Selective deletion of somitic tissues have established that only one of the somitic tissues is essential to axonal segmentation. The dermamyotome is irrelevant since both axons and neural crest cells advance in a segmental pattern even when dermamyotomes are absent (ref. 4, Fig. 4). In contrast, when sclerotome is removed, motor and sensory axons as well as neural crest cells that form sensory ganglia advance in a continuous, unsegmented pattern ${ }^{(6)}$. Furthermore, motor axons project specifically to limb muscles even in the complete absence of the segmental pattern of axon outgrowth ${ }^{(6)}$. Sclerotome populations are thus not essential for axon outgrowth, for neural crest migration, for sensory ganglion formation, or for the specificity of axonal projection to limb muscles. Sclerotome populations are essential only to the general, segmental patterns of axon and ncural crest advance. Axons form segmented spinal nerves and neural crest cells form segmented sensory ganglia as they encounter segmentally repeated tissues that function alternatively as paths (anterior sclerotome) and as barriers (posterior sclerotome).

Because the anterior sclerotome has been considered a favored path for axons and crest cells, it has been 
startling to find that the anterior sclerotome is further subdivided into a more dorsal path and a more ventral barrier. This functional subdivision is responsible for the positioning of the spinal nerves on the dorsalventral axis. During normal development, motor and sensory axons traverse the dorsal-anterior sclerotome but not the more ventral sclerotome that surrounds the notochord, the perinotochordal mesenchyme $e^{(6,7)}$ (Fig. 5). The barrier function of the perinotochordal mesenchyme was established by directly confronting outgrowing motor axons with perinotochordal mesenchyme $^{(8)}$. Motor axons were forced to initially extend more ventrally than normal by surgically rotating the developing spinal cord about its cross-sectional axis. When such axons contacted the perinotochordal mesenchyme, they turned to avoid it and traversed only the dorsal-anterior sclerotome.

Once axons have advanced through the dorsalanterior sclerotome of each somite and become segmented, they spread along the anterior-posterior axis within the unsegmented plexus mesenchyme at the base of the limb. The plexus region thus acts as a common pathway and its distal border is delineated by the adjacent pelvic girdle precursor which acts as a barrier at the limb base. In regions in which the girdle precursor is continuous along the dorsal-ventral axis, it bars axonal access to the limb. Discontinuities in the girdle precursor that contain plexus mesenchyme normally provide avenues for axons to enter the limb and form nerve trunks ${ }^{(7)}$ (Fig. 5). Axons readily



Fig. 5. Schematic diagram shows the spatial relations between paths (white) and barriers (stippling). Axons become spatially patterned along the anterior-posterior axis as they encounter segmentally repeated paths and barricrs, the anterior and postcrior sclcrotomes; they become spatially patterned along the dorsal-ventral axis as they encounter the path and barrier of the dorsal-anterior sclerotome and perinotochordal mesenchyme. Axons then spread along the anteriorposterior axis within a path tissuc, the plexus mesenchyme (indicated by dotted lines), which extends continuously along the anteriorposterior axis between the somites and the developing pelvic girdle. The pelvic girdle precursor is a barrier; axons penetrate it only through two holes that contain plexus mesenchyme. These holes delineate the positions where anterior and posterior nerve trunks form. Anterior is to the left; dorsal is up. traversed holes experimentally introduced into the girdle precursor ${ }^{(9)}$, showing that axons turn at the limb base in response to a barrier function of the pelvic girdle precursor rather than in response to properties of the plexus mesenchyme alone. Barrier function is as essential as path function to the establishment of the gross anatomical nerve pattern.

\section{Cellular Interactions That May Mediate General Guidance}

There is evidence that all three path tissues are paths because they share common features and that all three barrier tissues are barriers because they share distinguishing features. Each known barrier tissue has been suggested to express several molecular epitopes that are not expressed in paths ${ }^{(8)}$. Likewise, path tissues differentially cxpress butyrlcholinesterase and a $70 \mathrm{kd}$ membrane protein ${ }^{(10,11)}$ and are typified by widespread cell death and phagocytosis during axon outgrowth ${ }^{(12)}$. Moreover neural crest cells and motor, sensory and sympathetic preganglionic axons all independently respond to the three sets of path/barriers ${ }^{(8,13-17)}$, suggesting that the mechanisms of guidance are likely to be the same for a variety of invasive populations.

Before investing considerable effort in analyzing the molecular composition of tissues, it was important to determine whether path/barrier function could be due to the physical organization of these tissues rather than to their molecular composition per se. Physical barriers or guidance by channels have been shown to be unlikely by the simple expedient of looking with SEM and $\operatorname{TEM}^{(18,19)}$. There are no consistent physical differences between paths and barriers. Blood vessels or mats of extracellular material that could provide either physical barriers or preferred avenues of advance are similarly distributed in paths and barriers. There are no obvious channels or differences in cell density within paths that could make these tissues more easily penetrable.

Since differences in physical organization of paths and barriers are insufficient to explain their function, we can confidently begin to assess mechanisms on the cellular level. Path/barrier function could be due to the growth cones' response to: (1) a special property of paths, (2) a special property of barriers; (3) the contrast between the path and barrier environments, or to some combination of these factors. The cellular interactions that mediate the response of motor axons to anterior versus posterior sclerotome have been addressed in the most detail and provide support for three types of cellular interactions which fall neatly into each of these three classes.

(1) Paths may supply a diffusible, stimulatory substance. A preliminary report of a preferential orientation of motor axons toward anterior sclerotome cells in culture ${ }^{(20)}$ is in favor of such a chemotactic interaction.

(2) Barriers may contain a substance that actively inhibits growth cone motility. Contact paralysis is a well 
defined and obvious response to cell contact in which the growth cone collapses and transiently loses its ability to extend filopodia (see ref. 21). The reluctance of axons and neural crest cells to enter barrier environments could be explained if their motility were actively inhibited by contact with barrier cells. It is experimentally difficult, however, to discriminate between guidance by contact paralysis and a third potential mechanism, substratum preference.

(3) The combination of path and adjacent barrier may provide a contrast in substrata for axon and cell advance. Substratum preference is defined by the growth cone's ability to discriminate among different substrata that all support outgrowth to some degrec; when given a choice by direct contact with two substrata, the growth cone exhibits a preference by growing only on one substratum ${ }^{(22)}$. This is distinct from those guidance mechanisms in which a substratum is actively inhibitory or simply does not support outgrowth. In addition, proteolytic enzymes released by the growth cone could contribute to substratum preference, if paths were more susceptible to proteolysis and thus to growth cone penetration than were barriers (see 23).

Analyses of interactions between growth cones and anterior versus posterior sclerotome cells have provided evidence that is consistent with either substratum preference or contact paralysis. The carlicst analysis of interactions in culture showed that axons from the spinal cord (many of which should be motor axons) seldom extended on the surface of posterior sclerotome cells but often extended onto anterior sclerotome cells $^{(24)}$, a behavior in accord with either mechanism. The initial results from a tissue culture assay that retains the three-dimensional architecture and molecular features of sclerotome populations are likewise in favor of either mechanism, but additionally provide strong evidence against guidance by a totally non-permissive substratum, toxins, or a diffusible, repulsive molecule ${ }^{(20)}$

To distinguish between substratum preference and contact paralysis the cell interactions must be directly monitored over time. Initial reports of a study in which interactions between identified motoneurons and sclerotome populations were videotaped more strongly supports substratum preference ${ }^{(25)}$. Growth cones continue to enthusiastically extend processes when they contact posterior sclerotome cells but do not extend onto the surface of these cells; in contrast, growth cones readily traverse the surface of anterior sclerotome cells. Despite these results it is too early to rule out contact paralysis. For instance, as suggested by Davies et al. (26) (and see accompanying article by Davies and Cook, this issue), molccules that elicit paralysis may be nonuniformily localized on barrier cells and only inhibit individual filopodia that contact them. This would slow rather than stop the forward movement of growth cones and thus contribute to barrier function. This possibility can be systematically addressed by monitoring the rate of filopodial extension following contact with barrier versus path cells.

\section{Implications for Molecular Mechanisms of General Guidance}

The cellular interactions that may mediate path/barrier function each predict that different types of molecules are important and thus focus research toward particular classes of molecules. For instance, it is important to establish more fully whether or not the paths provide a diffusible cue. If so, it would be reasonable to next assess the possible contribution of known trophic molecules such as nerve growth factor. If not, then diffusible molecular candidates for path function could be ruled out.

A contact paralysis mechanism implies that there is a single ligand in all barrier tissues and a common receptor on all responding populations. The most likely candidate for a common ligand is a molecule that binds to peanut agglutinin lectin (PNA). Four lines of evidence implicate a PNA-binding molecule in barrier function and provide impetus for the current enthusiasm of several laboratories ${ }^{(26-28)}$ in isolating and characterizing such molecules: (1) PNA binds extensively to all known barriers but not to paths ${ }^{(29,30)}$; (2) PNA-binding and barrier function co-ordinately disappear in perinotochordal mesenchyme after notochord deletion $^{(8)}$; (3) PNA-binding correlates with putative barriers in the dermis ${ }^{(31)}$, roof plate (Oakley and Tosney, unpublished), somatosensory cortex ${ }^{(32)}$, and in oligodendrocytes $^{(33)}$; and (4) fractions isolated by PNAaffinity chromatography inhibit sensory growth cone advance in culture ${ }^{(26)}$.

Substratum preference is thought to be due to the cellular averaging of all the substratum contacts that a growth cone makes. A substratum preference mechanism therefore implies that all of the potential substratum molecules within paths and barriers could contribute to guidance function. Since molecules that are known to be excellent substrata are present in both paths and barriers ${ }^{(8)}$, it is not likely that barriers are totally non-permissive substrata; it is more likely that barriers provide poor substrata only in comparison with paths. Several molecules typical of carly cartilage differentiation that are known to provide relatively poor substrata in culture are differentially expressed in barriers (see ref. 8). PNA-binding is a marker for early cartilage differentiation as well, and a PNA-binding molecule may contribute to a substratum preference mechanism rather than to a contact paralysis mechanism. If substratum preference is shown to be important to guidance, the molecular mechanism could be more directly addressed. For instance, what is the hierarchy of growth cone preference for the various molecular substrata that are expressed in paths and barriers? Do some molecules typical of barriers mask molecular binding sites that would otherwise provide more preferred substrata? Are some components of paths 
more susceptible to proteolytic enzymes released by growth cones?

\section{Independence and Co-ordination of General and Specific Cues}

The guidance of the epaxial motoneurons provides an example of the integration of general and specific cues during axon outgrowth. In addition to their directional response to target, the epaxial motor axons obey the constraints of path/barrier environments ${ }^{(4)}$. For instance, axons deprived of a target in their own segment do not take the most direct route to a target in a more posterior segment; they take a route that avoids the posterior sclerotome (e.g., segment 4 in Fig. 4). Despite the coordinate response of epaxial motor axons to the two types of cues, the specific response is independent of the general guidance cues. When sclerotome is completely removed, outgrowth of epaxial motor axons is still target-dependent and axons then take the most direct route to the nearest target ${ }^{(6)}$. Since epaxial and other axons can respond independently to general and to specific cues ${ }^{(6,34)}$, it is likely that different molecular species mediate general and specific guidance.

Even if different molecules subserve general and specific guidance functions, both functions may be mediated by the same classes of cellular interactions. For instance, chemotaxis could mediate both specific and general guidance of epaxial motor axons. A general stimulatory cue could help assure that epaxial and other populations colonize dorsal-anterior sclerotome rather than posterior sclerotome or perinotochordal mesenchyme; a diffusible cue that was specifically sensed by epaxial growth cones could assure that these motor axons extend dorsally toward epaxial muscle rather than laterally toward the limb base as they traverse the dorsal-anterior sclerotome.

A contact-paralysis interaction could mediate specific target recognition as well as barrier function. For instance, a molecule on the surface of epaxial muscle could cause paralysis of epaxial but not other growth cones and help stabilize specific contacts. In fact, the epaxial growth cones assume a morphology reminiscent of paralyzed growth cones upon contact with epaxial muscle in vivo ${ }^{(35)}$.

Likewise, a substratum preference mechanism could mediate both specific and general guidance. This possibility is in accord with evidence that paths provide substrata that are by no means optimal; general paths may be near the bottom of any hierarchy of substratum preference. For instance, even though motor growth cones consistently prefer anterior over posterior sclerotome as a substratum in culture, they grow more rapidly on laminin or on muscle cells than on either sclerotome population ${ }^{(20)}$. This means that any molecule that provides a better substratum for a particular population could provide a specific cue for its outgrowth. As a speculative example, epaxial but not limb motor axons might have receptors for a molecule secreted by dermanyotome which binds in gradientfashion to cells or ECM and thus orients their outgrowth.

\section{Conclusions}

It is clear that a number of cellular interactions may mediate the axonal responses to both general and specific cues. Detailed analysis of the interactions between growth cones and the relevant populations in culture is essential to define the relevant interactions and to implicate the most likcly classes of molecules. Since embryonic surgeries have identified the relevant tissues, tissue culture and biochemical approaches are now possible. Embryonic surgerics have thus provided a requisite foundation for an understanding of the mechanisms of motor axon guidance in the chick embryo.

\section{Acknowledgements}

Work in the author's laboratory is supported by NIH grants NS-21308 and NS-27634. Thanks are duc to Robert Oakley for useful discussion.

\section{References}

1 LANDMESSF, L. T. (1985). The development of specific motor pathways in the chick embryo. Trends Neurosci. 7, 336-339.

2 Lance-Ionfs, C. I. and Landmfssfr, L. T. (1981). Pathway selection by embryonic chick motoneurons in an experimentally altered environment. Prox. $R$ Soc. London $B, 214,19-52$.

3 LaNCE-JONES, C. J. (1988). Motoneuron axon guidance: Development of specific projections to two muscles in the embryonic chick limb. Brain Behav. Evol. 31, 209-217.

4 Tosnfy, K. W. (1987). Proximal tissues and patterned neurite outgrowth at the lumbusacral level of the chick embryo: Deletion of the dermamyotome Devl Biol. 122, 540-588.

5 Keynes, R. J. AND Stern, C. D. (1984). Segmentation in the vertebrate nerwous system. Nature 310, 786-789.

6 TosNFy, K. W. (1988). Proximal tissues and patterned neurite outgrowth at the lumbosacral level of the chick embryo: Partial and complete deletion of the somite. Devl Biol. 127, 266-286.

7 Tosnex. K. W. AND LANDMESSER, L. T. (1985). Development of the major pathways for neurite outgrowth in the chick hindlimb. Dew. Biol. 109, 193-214.

8 Tosnfy, K. W. ANd OAKLey, R. A. (1990). Perinotochordal mesenchyme acts as a barrice to axon advance in the chick embryo: implications for a general mechanism of axon guidance. Exp. Neurol. 109, 75-89.

9 Tosney, K. W. and Landmesser, L. T. (1984). Pattern and specificity of axonal outgrowth following varying degrees of chick limb bud ablation. $J$. Nevtosct. 4, 2158-2527.

10 LAYER, P. G., AlBER, R. AND RATHJEN, F. G. (1988). Sequential activation of butyrul-cholinesterase in rostral half somites and acelylcholinesterase in motoneurons and myotomes preceding growth of motor axons. Development $102,387-396$.

11 TanaKa, H., Agnta, A. and Obata, K. (1989), A new membrame antigen revcalcd by monoclonal antibodies is associated with motoneuron axonal pathways. De:l Biol. 132, 419-435.

12 Tosnfy, K. W. Schroeter, S. and Pokrzywinski, J. A. (1988). Cell death delineates axon pathways in the hindlimb and does so independently of neurite outgrowth. Devl Biol. 130, 558-572.

13 Rickmann, M., Faucet, J. and Keynes. R. (1985). The migration of neural crest cells and the growth of motor axons through the rostral half of the chick somite. J. Embryol. Exp. Morphol. 90, 437-455.

14 Bronner-Fraser. M. (1986). Analysis of the early stages of trunk neural crest migration in avian embryos using monoclonal antibody HNK-1. Devl Biol. $115,44-55$

15 Loking, J. F. AND ERICKSON, C. A. (1987). Neural crest cell migratory pathways in the chick embryo. Devl Biot. 121,230-236. 
16 Newgreen, D. Schfer, M. and Kasiner, V. (1986). Morphogenesis of sclerotome and neural crest in avian cmbryos: In vivo and in vitro studies on the role of notochordal extracellular material. Cell Tiss Res. 244, 299-313

17 LANDMLSSLR, L. AND HONTG, M. G. (1986). Altered sensory projections in the chick hind limb following the early removal of motoncurons. Devi Biol. 118 $5) 1-531$

18 Tosney, K. W. (1988). Somites and axon guidance. Scan. Microsc. 2 $427-442$.

19 Dehingostle, D. and Tosney, K. W. (1990). Initial axon outgrowth. Soc Neuro. Abs, 16, 1006 .

20 OAKLEY, R. A. AND TOSNEY, K. W, (1989). Analysis of the cellulat interactions that mediate the development of motor axon scgmentation. Soc Veuro. Abs. 15, 875 .

21 Kaptilamiar, J. P. and RAper, J. A. (1987). Collapse of growth cone structure on contact with specific neurites in culture. $J$. Neurosci. 7, 201-213. 22 Letourreau, P. (1975). Cell-to-substrate adhesion and guidance of axona elongation. Devl Biol. 44, 92-101.

23 Pitrman, R. N. (1985). Release of plasminogen activalor and a calciumdependent metalloprotease from cultured sympathetic and sensory neurons. Jey Biol. 110, 91-101.

24 Stern, C. D., Sisodiya, S. M. and Kfynfs, R. J. (1986). Interaction between neurites and somite cells: Inhibition and stimulation of nerve growth in the chick embryo. I. Embryol. Exp. Morphol. 91, 209-226.

25 Tosney, K. W. (1987). Growth cone interaclions with anterior and posterior sclerotome. Soc. Neuro. Abs. 13, 253.

26 Davies, J. A., Cook, G. M. W., Stern, C. D. And Kernes, R. J. (1990) Isolation from chick somites of a glycoprotcin fraction that causes collapse of dorsal root ganglion growth cones. Neuron 2, 11-20.

27 Hormas, S. Crossin, K. L. ann Eibjimay, G. M. (1988). Molecular forms, binding functions, and developmental expression patterns of cytotactin and cytotactin-binding proteoglycan, an interactive pair of extracellular matrix molccules $J$. Cell Biol. 106, 519-532.

28 BONHOEFHER, F. (1989). Inhibitory influences on growth cones and cells. Soc. Neuro. Abs. 15, 1106.

29 OAkify, R. A And Tosnfy, K. W. (1988). Peanut agglutinin (PNA) binds to tissues that act as barriers to axon advance in the chick embryo. Soc. Neuro. Abs. 14,870

30 OAKIFY, R. A. AND Toshey, K. W. (1990). Evidence for the delineation of axon pathways by inhibitory boundaries. Soc. Neuro. Abs. 16, 1006.

31 Sciroeter, S., Lasky, C. OAkldy, R. A. AND Tosney, K. W. (1990) Evidence for the delineation of neural crest migration pathways by inhibitory boundaries. Soc Newro. Abs. 16, 313 .

32 STeindlek, D. A. ANT COOPER, N. G. F. (1987). Glial and glycoconjugate boundarics during postnatal development of the central nervous system. $D e v$ Brain Res. 36. 27-38.

33 Mikot, D. D. AND Stefanssov, K. (1988). A phosphatidylinositol-linked peanut agglutinin-binding glycoprotein in central nervous systcm myclin and on ooligodendrocyles. J. Cell Biol. 106, 1273-1279.

34 TOSNEY, K. W. AND I IAGEMAN, M. (1989). Different subsets of axuna guidance cucs are essential for sensury neurite outgrowth to cutaneous and muscle largets in the dorsal ramus of the embryonic chick. $J$. Exp. Zool. 251 $232-244$.

35 Tosney, K. W. and Landmessfr, I. T. (1985). Growth cone morphology and trajectory in the lumbosacral region of the chick cmbryo. J. Neurosci. 5 $345-2358$.

Kathryn W. Tosney is at the Biology Department, The University of Michigan, Ann Arbor, MI 48109 , USA.

\section{Correspondence}

BioEssays welcomes correspondence. Readers wishing to comment on subjects or issues raised in our pages should send their letters either to the editor, Dr Adam S. Wilkins, Company of Biologists, Department of Zoology, University of Cambridge, Downing St., Cambridge CB2 3EJ, UK or to the chairman of the editorial board, Dr Kermit L. Carraway, Department of Cell Biology and Anatomy, PO Box 016960, University of Miami School of Medicine, Miami, FL 33101, USA. 